\title{
Path Integral Optimization as Circuit Complexity
}

\author{
Hugo A. Camargo, ${ }^{1,2, *}$ Michal P. Heller, ${ }^{1, \dagger}$ Ro Jefferson, ${ }^{1, \ddagger}$ and Johannes Knaute ${ }^{1,2, \S}$ \\ ${ }^{1}$ Max Planck Institute for Gravitational Physics (Albert Einstein Institute),Am Mühlenberg 1, 14476 Potsdam-Golm, Germany \\ ${ }^{2}$ Department of Physics, Freie Universität Berlin, Arnimallee 14, 14195 Berlin, Germany
}

(Received 29 April 2019; published 1 July 2019)

\begin{abstract}
Early efforts to understand complexity in field theory have primarily employed a geometric approach based on the concept of circuit complexity in quantum information theory. In a parallel vein, it has been proposed that certain deformations of the Euclidean path integral that prepare a given operator or state may provide an alternative definition, whose connection to the standard notion of complexity is less apparent. In this Letter, we bridge the gap between these two proposals in two-dimensional conformal field theories, by explicitly showing how the latter approach from path integral optimization may be given by a concrete realization within the standard gate counting framework. In particular, we show that, when the background geometry is deformed by a Weyl rescaling, a judicious gate counting allows one to recover the Liouville action as a particular choice within a more general class of cost functions.
\end{abstract}

DOI: 10.1103/PhysRevLett.123.011601

Introduction.-One of the most interesting developments of the past decade is the confluence of ideas from quantum gravity, quantum field theory (QFT), and quantum information science. The impact of this research trend is perhaps most striking in holography (AdS/CFT) [1-3] where, e.g., the entanglement entropy of spatial subregions in the boundary field theory has been shown to play a prominent role in describing higher-dimensional (from this perspective, emergent) geometries; see Ref. [4] for a review. While the entanglement entropy is represented by extremal codimension-2 surfaces in the bulk [5-9], it has been conjectured that certain codimension-1 [10] and codimension- $0[11,12]$ bulk objects are related to the notion of "complexity" in the dual QFT. In particular, these objects appear to be natural probes of the black hole interior in AdS/CFT, the reconstruction of which is a timely problem of clear importance for our efforts to understand quantum gravity [13-18].

These so-called holographic complexity proposals have not only contributed to ongoing research on the emergence of dynamical spacetimes from microscopic quantum mechanical degrees of freedom, but have also motivated the exploration of complexity in QFTs. The concept of complexity originates in quantum computing and is based on approximating a given (typically unitary) operator in terms of some fundamental building blocks. In the context of quantum circuit design, the latter correspond to the gates

Published by the American Physical Society under the terms of the Creative Commons Attribution 4.0 International license. Further distribution of this work must maintain attribution to the author(s) and the published article's title, journal citation, and DOI. Funded by SCOAP . used in constructing circuits that realize the given operation, the complexity of which is defined as the minimumlength circuit that achieves this goal. Note that, by fixing a reference state, this same framework allows one to speak of the complexity of states in the theory by optimizing over all circuits (operators) that produce the desired target state. From this perspective, the complexity of operators is the more elementary notion, and it is this question on which we will focus in the present Letter.

In practice, performing the optimization over all possible constructions is a difficult task. To surmount this problem in the original context of quantum circuit design, Nielsen et al. [19-21] developed a procedure by which one could apply variational calculus to this optimization problem by associating a geometry to the space of circuits based on the algebra of gates. Inspired by this approach, Jefferson and Myers [22] introduced the notion of circuit complexity in QFT by finding geodesics in the space of Gaussian states. Simultaneously, Chapman et al. [23] put forth a complementary proposal based on continuous tensor network ideas [24-26]. Together with subsequent developments [27-34], these may be collectively considered as a geometric approach to complexity based on quantifying fundamental operations (i.e., gates). We shall refer to this line of thinking rather broadly as "circuit complexity," as per [22].

A second main approach based on so-called path integral optimization has been proposed in [35-37]. This was based on the observation that if one discretizes the Euclidean path integral, which prepares a given state over a flat background, then a multiscale entanglement renormalization ansatz [38] can be loosely related to an optimization of this network, which corresponds to performing the path integral over a Weyl-rescaled geometry. The resulting change in the path integral-namely, the well-known Liouville action 
introduced below-was then defined as the complexity of the corresponding state. This idea has since been made somewhat more precise in [39,40]; see also [41] for a further development based on this prescription. However, in addition to certain technical subtleties, a conceptual drawback of this proposal is that it is not obvious why the Liouville action should be related to complexity in the usual sense of the word.

The aim of the present Letter is to explicitly demonstrate that path integral optimization in its best-explored setting of two-dimensional conformal field theories (CFTs) may be given a precise formulation in terms of circuit complexity.

Review of approaches to complexity in QFT. - Let us begin by briefly reviewing the geometric approach introduced in Refs. [22,23]. In this approach, one represents the circuit-i.e., the operator $V$-as a path-ordered exponential, consisting of a sequence of elementary "gates" generated by $\mathcal{O}_{I}$,

$$
V=\mathcal{P} \exp \left(-\int_{0}^{\lambda} d \kappa \sum_{I} Y^{I}(\kappa) \mathcal{O}_{I}\right)
$$

In this expression, one can think of $\kappa$ as parametrizing the infinitesimal layers of the circuit and $Y^{I}$ as controlling the gates inserted at that layer; in geometrical language, the latter may be regarded as a velocity component along the path $\kappa$. In order to measure the circuit length, one defines a cost function $\mathcal{D}$, in which choice one has a considerable amount of freedom. For example, the choice most directly related to counting gates is the $L^{1}$ norm, $\mathcal{D}_{1}=$ $\int_{0}^{\lambda} d \kappa \sum_{I}\left|Y^{I}\right|$. If one instead uses the $L^{2}$ norm $\mathcal{D}_{2}=$ $\int_{0}^{\lambda} d \kappa \sqrt{\sum_{I J} \eta_{I J} Y^{I} Y^{J}}$ with $\eta_{I J}$ some positive-definite matrix, then one arrives at the familiar problem of finding geodesics on a Riemannian manifold; see [22] and related work above for details. Of course, there are other norms motivated from quantum information theory that serve equally well, in particular, the Fubini-Study distance used in [23].

We now turn to the path integral approach, in preparation to reframe the latter in the language of circuit complexity. We focus here on a specific example pioneered in Ref. [35], in which one works with an unnormalized thermal density matrix

$$
\rho_{\beta}=\exp (-\beta H)
$$

where $H$ is the Hamiltonian operator of a two-dimensional CFT on a line. We regard $\rho_{\beta}$ as an operator $V$ (defined up to an overall normalization), rather than state, which we wish to decompose as in Eq. (1). This particular operator is of course very interesting since, in addition to representing a thermal state in the CFT, in theories with a mass gap it also acts as a projector onto the vacuum state in the limit $\beta \rightarrow \infty$.
The matrix elements of $\rho_{\beta}$ may be represented via a Euclidean path integral over a strip of flat geometry,

$$
d s_{0}^{2}=d \tau^{2}+d x^{2},
$$

with width $\beta$ in the $\tau$ direction,

$$
\left\langle\phi(\beta, x)\left|\rho_{\beta}\right| \phi(0, x)\right\rangle=\int_{\hat{\phi}(0, x)=\phi(0, x)}^{\hat{\phi}(\beta, x)=\phi(\beta, x)} \mathcal{D} \hat{\phi} e^{-S_{\mathrm{CFT}}[\hat{\phi}]},
$$

where $\hat{\phi}$ denotes the fields in the theory and $\phi$ are particular eigenstates of field operators. It then transpires that if one deforms the background geometry over which the path integration is performed by a Weyl factor $\omega(\tau, x)$,

$$
d s_{\omega}^{2}=g_{\mu \nu} d x^{\mu} d x^{\nu}=e^{2 \omega(\tau, x)}\left(d \tau^{2}+d x^{2}\right),
$$

such that $\omega(\tau=0, x)=\omega(\tau=\beta, x)=0$, then the same operator $V$ is prepared (again up to an overall normalization), cf. Fig. 1. As alluded in the Introduction, Refs. [36,37] then proposed that a natural cost function to consider is the Liouville action

$$
S_{L}=\frac{c}{24 \pi} \int_{0}^{\beta} d \tau \int_{-\infty}^{\infty} d x\left[\Lambda e^{2 \omega}+\dot{\omega}^{2}+\omega^{\prime 2}\right]
$$

where ${ }^{\circ}$ and ' denote derivatives with respect to $\tau$ and $x$, respectively, and $c$ is the central charge. The Liouville action governs the change in the path integral measure under the above Weyl rescaling. Here, $\Lambda \sim \epsilon^{-2}$, where $\epsilon$ denotes some UV cutoff in real space (i.e., the lattice spacing).

In the present Letter, we will demonstrate that, up to an unimportant overall normalization, $S_{L}$ corresponds in a precise way to a particular cost function in the geometric approach to circuit complexity outlined above. Before proceeding, let us stress two aspects of the Liouville action that will be relevant later. The first is that the Liouville

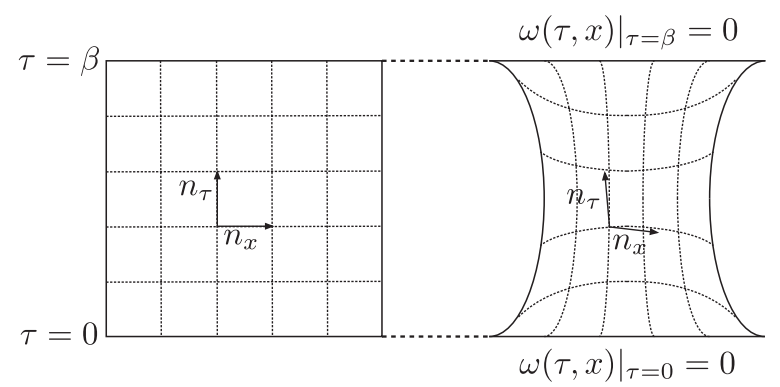

FIG. 1. Euclidean strip over which we perform the path integral (4). The left image represents the flat geometry (3), while the right represents the Weyl-rescaled background (5). Note that we hold the boundary conditions at $\tau=0$ and $\tau=\beta$ fixed. This guarantees that we produce the same operator $\rho_{\beta}$, up to an overall normalization governed by the exponent of the Liouville action (6). 
action is properly a covariant expression, which in arbitrary coordinates $\xi^{\mu}$ can be shown to take the form [42]

$$
S_{L}=\frac{c}{24 \pi} \int d^{2} \xi \sqrt{g}\left[\Lambda+\frac{1}{4} \partial_{\mu} \chi \partial^{\mu} \chi\right],
$$

where the scalar $\chi$ is a covariant albeit nonlocal expression defined in terms of the Ricci scalar $R$ and the propagator $\square^{-1}$ for a massless scalar field,

$$
\chi(\xi)=\int d^{2} \tilde{\xi} \sqrt{g(\tilde{\xi})} \square^{-1}(\xi, \tilde{\xi}) R(\tilde{\xi}) .
$$

Furthermore, we impose that $\chi$ vanishes at the boundaries to make it unique. Note that by changing the relative normalization between the $\dot{\omega}^{2}$ and $\omega^{\prime 2}$ terms in Eq. (6), one would not be able to recover the covariant form (7). The second comment is that one should regard the Liouville action as consisting of the two leading terms in the expansion in derivatives of the metric weighted with respect to $\epsilon$ : the cosmological constant term $\Lambda$, which is $\mathcal{O}\left(\epsilon^{-2}\right)$ and diverges in the continuum, is related to the energy-momentum tensor renormalization, while the nonlocal term is $\mathcal{O}\left(\epsilon^{0}\right)$ and gives rise to the trace anomaly. One could add terms having more derivatives, but they would necessarily come with positive powers of $\epsilon$ and hence vanish in the $\epsilon \rightarrow 0$ limit. While these terms might become important for the path integral optimization program, as we discuss below, we ignore them in the present Letter.

Euclidean path integrals as circuits.-We now wish to interpret the Euclidean path integral on the Weyl-rescaled geometry as a circuit in the manner of Eq. (1). To do so, we will make use of a formula developed in Refs. $[39,40]$ in the context of tensor networks linking path integrals on curved geometries with the exponentiation of the energy-momentum tensor components of two-dimensional CFTs in Minkowski space.

The relevant statement is the following. If one considers a Euclidean path integral for a $\mathrm{CFT}_{2}$ on a generic background in coordinates in which constant Euclidean time $t$ slices are flat lines, i.e.,

$d s^{2}=\left[a(t, y)^{2}+b(t, y)^{2}\right] d t^{2}+2 b(t, y) d t d y+d y^{2}$,

then, up to an unimportant normalization, this path integral computes the matrix elements of the operator

$V=\mathcal{P} \exp \left(-\int_{t_{i}}^{t_{f}} d t \int d y[a(t, y) h(y)+i b(t, y) p(y)]\right)$,

where $h \equiv T_{t_{M} t_{M}}$ and $p \equiv T_{t_{M} y}$ are given in terms of the stress tensor in Minkowski space, whose line element we write as $-d t_{M}^{2}+d y^{2}$, and the path ordering is applied to the integration over the Euclidean time $t$. One can understand

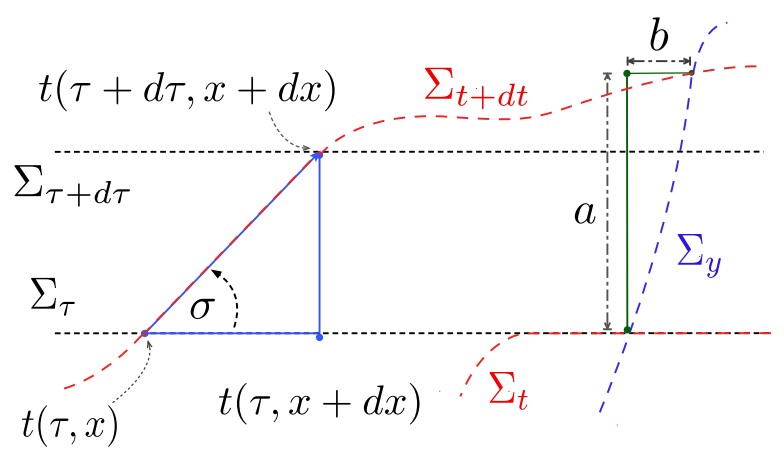

FIG. 2. $\Sigma_{\tau}$ and $\Sigma_{t}$ denote time slices of the background geometry in $(\tau, x)$ coordinates [see Eq. (5)] and $(t, y)$ coordinates [see Eq. (9)], respectively. At a given point, the Euclidean angle between tangents to $\Sigma_{t}$ and $\Sigma_{\tau}$ is given by the function $\sigma(\tau, x)$, as defined in Eq. (12). On the right side of the figure, $a$ and $b$ appear as defined by Eq. (9), which illustrates their geometrical interpretation.

this expression pictorially in a very simple way, as illustrated in Fig. 2.

We then observe that Eq. (10) has the same form as our circuit (1). Accordingly, we interpret the path integral (10) as a bilinear map that acts on the Hilbert space on a slice of Euclidean time $t$. Comparing with Eq. (1), the circuit parameter $\kappa$ is then identified with $t$, and the generators of the gates $\mathcal{O}^{I}$ are the energy-momentum components on a slice of flat Minkowski space, namely, the Hermitian and anti-Hermitian operators $h$ and ip, respectively. We do not include the pressure $T_{y y}$ since the tracelessness of the energy-momentum tensor makes it equal, as an operator, to the energy density $h$. The index $I$ runs over the full range of $y$ and differentiates between $h$ and $i p$. Note that, in contrast to the existing literature on circuit complexity reviewed above, we are working with circuits consisting of both Hermitian (Euclidean) and unitary transformations. This is a natural consequence of the fact that the Wick rotation to Euclidean time places the $t t$ and $t y$ components of the stress tensor on different footing.

To proceed, we must identify coordinate transformations from the $(\tau, x)$ coordinates in the Weyl-rescaled form of the metric (5) to the $(t, y)$ coordinates (9). The most general coordinate transformation can be parametrized by a diffeomorphism $t(\tau, x), y(\tau, x)$. In Appendix $\mathrm{A}$ of the Supplemental Material [43] we develop some intuition for the particular case where only $y=y(\tau, x)$ is transformed. One can then solve for $a$ and $b$ in terms of $t$ and $y$, which leads to the following result:

$$
a=\frac{\dot{t} y^{\prime}-t^{\prime} \dot{y}}{\dot{t}^{2}+t^{\prime 2}} \quad \text { and } \quad b=-\frac{\dot{t} \dot{y}+t^{\prime} y^{\prime}}{\dot{t}^{2}+t^{\prime 2}} \text {. }
$$

Of course, one also needs to ensure that $y(0, x)=y(\beta, x)=$ $x$ and that $t(0, x)$ and $t(\beta, x)$ are constant, as per the boundary conditions explained above. In this most general 
case, we have the freedom to choose two functions subject to the aforementioned boundary conditions. It is important to note that, while we think of $a$ and $b$ as functions specifying the circuit, a generic choice of these functions will not return a circuit of the form (10) that represents, up to normalization, the operator $\rho_{\beta}$ (2). However, the specific parametrization (11) guarantees this.

Before we proceed to specifying the cost function, it will be very convenient to introduce an angle $\sigma$, defined as

$$
t^{\prime}=-\dot{t} \tan \sigma
$$

the intuition for which is explained in Fig. 2. Furthermore, we note that derivatives of $t$ and $y$ are related to the Weyl factor $\omega$ by

$$
y^{\prime}=e^{\omega} \sec \sigma-\dot{y} \tan \sigma .
$$

In what follows, we will count gates in terms of cost functionals of $a, b$ (11); since our goal is to reproduce the Liouville action naturally expressed in terms of the Weyl parameter, we will aim to reexpress these cost functionals in terms of $\omega$ and the free (gauge) parameter $\sigma$.

Cost functions and the Liouville action.-In counting gates, our point of departure is regarding Eq. (10) as a circuit of the form (1). Of course, as in previous works on the geometric approach to circuit complexity, there are many cost functions one could consider. For example, a natural choice is an $L^{1}$ norm; while this is ultimately not the choice we wish to make, it is helpful for gaining some intuition about the cost function, and we refer the reader to the Appendix B (including Refs. [44-46]) for a discussion of this case and Appendix C of the Supplemental Material [43] for the $\kappa=2$ norm as another common, albeit problematic choice of the cost function. A further choice is the more familiar $L^{2}$ norm, but this is rather more subtle than the $L^{1}$ norm, since this puts the integral over $y$ under the square root, and hence does not lead to natural expressions in terms of $\omega$ and $\sigma$ as functions of $\tau$ and $x$. Furthermore, if we wish to obtain the Liouville action as a proper cost function, it appears that the most appropriate choice lies between these two familiar norms and takes the form

$\mathcal{D}_{L} \simeq \int d t d y \frac{1}{\epsilon^{2}} \sqrt{a^{2}+\epsilon^{2} \eta_{(\partial a)^{2}}\left(\partial_{y} a\right)^{2}+\epsilon^{2} \eta_{(\partial b)^{2}}\left(\partial_{y} b\right)^{2}+\cdots}$,

where the ellipsis again denotes higher derivative terms that we shall drop, and we have neglected more complicated terms of the sort discussed above. As alluded to above, treating this expression exactly does not result in a diffeomorphism-invariant (i.e., $\sigma$ independent) cost function. However, if we choose equal penalty factors, i.e., $\eta_{(\partial a)^{2}}=\eta_{(\partial b)^{2}}$, and expand to next-to-leading order in $\epsilon$, we find that $\omega$ and $\sigma$ decouple, and our cost function takes the form

$$
\begin{aligned}
\mathcal{D}_{L} \simeq & \int d \tau d x\left\{\frac{e^{2 \omega}}{\epsilon^{2}}+\frac{1}{2} \eta_{(\partial a)^{2}}\left(\dot{\omega}^{2}+\omega^{\prime 2}\right)\right. \\
& \left.+\frac{1}{2} \eta_{(\partial a)^{2}}\left(\dot{\sigma}^{2}+\sigma^{\prime 2}\right)+\eta_{(\partial a)^{2}}\left(\omega^{\prime} \dot{\sigma}-\dot{\omega} \sigma^{\prime}\right)+\cdots\right\} .
\end{aligned}
$$

Observe that the last term to this order is a total derivative, and hence the optimization over $\omega$ is independent of the choice of $\sigma$. Furthermore, the $\omega$-dependent part is none other than the Liouville action. This implies that the Liouville action that underlies the approach to complexity from "path integral optimization" can be given a precise interpretation as an approximation to a particular choice of cost function. However, from this perspective there appears nothing fundamental about the Liouville action as a measure of complexity. Indeed, the former simply arises from the change in the path integral measure under Weyl rescaling, as reviewed above, and we have relied on a degree of fine-tuning of the cost function in order to recover a complexity measure of the same form. A similar observation was made in [47], where the Liouville action was found to be the next-to-leading-order term in an $\epsilon$ expansion of a bulk gravitational action with a boundary term in three-dimensional anti-de Sitter space with a position-dependent cutoff.

As a closing remark, we note that for $\sigma=0$ the cost function (14) resembles a Dirac-Born-Infeld (DBI) action (for a review, see [48]) for the field $\omega$, and hence in terms of the field $\chi$ defined in Eq. (8), we may write

$$
\mathcal{D}_{\mathrm{DBI}} \simeq \int d^{2} \chi \sqrt{\operatorname{det}\left(g_{\mu \nu}+\epsilon^{2} \partial_{\mu} \chi \partial_{\nu} \chi\right)} .
$$

It would be interesting to pursue this analogy further, but we leave this for future work.

Discussion and outlook.-This Letter can be regarded as an attempt to define circuit complexity in QFT as a direct functional of sources appearing in the underlying Euclidean path integral. Furthermore, as opposed to the earlier works on gate complexity in QFT, we explicitly use only local gates. In this framework, we were able to show that the cost function appearing in the path integral optimization approach, namely, the Liouville action given by Eq. (7), can be thought of as an approximation to a genuine gate counting procedure in which the source is the background metric.

One aspect to emphasize is that the covariance with respect to an underlying metric is lost for a genuine cost function, since these are defined with respect to some time foliation. So far we were only able to recover covariance approximately to next-to-leading order in the UV cutoff in 
the relevant expansion for obtaining the Liouville action, cf. Eq. (15). In general, one expects that covariance combined with a proper gate counting prescription poses quite restrictive constraints on allowed metric functionals. On this front, note that, in the path integral optimization approach, one just varies the cost function with respect to $\omega$. However, in the covariant approach, one would also vary with respect to other metric components, and this should lead to some constraint equations.

Another important item that we want to stress in the context of Liouville is that our cost functions are not sensitive to the central charge of the CFT. Of course, one has the freedom to introduce the central charge in the overall normalization (or in penalty factors), but the arguments above do not provide any physical justification for doing so, and consequently, we have only considered cost functions defined up to some overall prefactor.

There are two further implications of this Letter we wish to highlight. First, a proof of the holographic complexity proposals should occur through the equality of bulk and boundary Euclidean partition functions, and the interface between the two sides is governed by the boundary (QFT) sources. While there have already been several attempts in this general direction [49-52], the present Letter is perhaps the first to construct genuine cost functions for a particular class of source configurations. Second, in the path integral optimization program [35-37], one optimizes the Liouville action alone, i.e., without including higher-order corrections in $\epsilon$. Since we found it rather nontrivial to construct a cost function that formally matches Liouville, while the seemingly more natural ones included higher-order corrections in $\epsilon$, one should keep in mind that perhaps the optimal circuits or geometries for genuine cost functions might be different than the ones obtained using the Liouville action alone. On the other hand, the latter were found to correspond to geometries consistent with hyperbolic spacetimes; in light of the complexity $=$ volume proposal, one may therefore wish to keep this feature as an additional condition beyond covariance and gate counting. In this spirit, Eq. (16) might be taken as an interesting point of departure for studies of cost functions to all orders in $\epsilon$.

Regarding direct extensions of this work, it would be very interesting to find a cost function that is fully covariant to all orders in $\epsilon$. Furthermore, an approach closely related to the use of the Fubini-Study distance has been developed for unitary transformations with energy-momentum tensor insertions in [53], building on earlier results in [54]. Although [53] focused on purely holomorphic or antiholomorphic transformations, it seems important to understand the relation with the present work in greater detail. One attempt in this direction might be to use our circuits contracted with some sample state (for example, the family of states used in [53]) and in this way calculate the FubiniStudy distance along the lines of Ref. [23]. Furthermore, when compensating for the derivative dimensions in the present Letter, we relied on the UV regulator $\epsilon$ as the only obvious scale in the problem. However, if one has insertions of operators at different spatial positions on a constant $t$ slice, say at $y$ and $\tilde{y}$, then the Euclidean distance between them acts as another relational dimensionful quantity. Additionally, the current approach may be generalized to provide a definition of complexity for mixed states by including nonunitary transformations; $\mathrm{cf}$. $[29,55]$ for earlier works in this vein.

We wish to thank E. Brehm, P. Caputa, B. Czech, M. Flory, B. Freivogel, L. Hackl, D. Hofman, L. Hadasz, R. Janik, J.-L. Lehners, T. Osborne, V. Schomerus, V. Svensson, S. Theisen, T. Takayanagi, and $\mathrm{H}$. Wang for helpful discussions and correspondence and, especially, J. Hung for her key insight inspired by DBI actions that allowed us to derive the Liouville action as an approximation to a cost function. We are also very grateful to D. Das and S. He for their input during earlier stages of the project. Our special thanks go also to S. Singh, M. Walter, and F. Witteveen, as well as J. de Boer, S. Chapman, and I. Reyes for their feedback and collaboration with some of us on related topics. The Gravity, Quantum Fields, and Information group at AEI is generously supported by the Alexander von Humboldt Foundation and the Federal Ministry for Education and Research through the Sofja Kovalevskaja Award. H. C. is partially supported by the Konrad-Adenauer-Stiftung through their Sponsorship Program for Foreign Students.

*hugo.camargo@aei.mpg.de

On leave from National Centre for Nuclear Research, Pasteura 7, 02-093 Warsaw, Poland.

michal.p.heller@aei.mpg.de

rjefferson@aei.mpg.de

\$johannes.knaute@aei.mpg.de

[1] J. M. Maldacena, Int. J. Theor. Phys. 38, 1113 (1999); Adv. Theor. Math. Phys. 2, 231 (1998).

[2] E. Witten, Adv. Theor. Math. Phys. 2, 253 (1998).

[3] S. S. Gubser, I. R. Klebanov, and A. M. Polyakov, Phys. Lett. B 428, 105 (1998).

[4] M. Rangamani and T. Takayanagi, Lect. Notes Phys. 931, 1 (2017).

[5] S. Ryu and T. Takayanagi, Phys. Rev. Lett. 96, 181602 (2006).

[6] V. E. Hubeny, M. Rangamani, and T. Takayanagi, J. High Energy Phys. 07 (2007) 062.

[7] H. Casini, M. Huerta, and R. C. Myers, J. High Energy Phys. 05 (2011) 036.

[8] A. Lewkowycz and J. Maldacena, J. High Energy Phys. 08 (2013) 090.

[9] X. Dong, A. Lewkowycz, and M. Rangamani, J. High Energy Phys. 11 (2016) 028.

[10] L. Susskind, Fortschr. Phys. 64, 44 (2016); 64, 24 (2016).

[11] A. R. Brown, D. A. Roberts, L. Susskind, B. Swingle, and Y. Zhao, Phys. Rev. Lett. 116, 191301 (2016). 
[12] A. R. Brown, D. A. Roberts, L. Susskind, B. Swingle, and Y. Zhao, Phys. Rev. D 93, 086006 (2016).

[13] B. Freivogel, R. A. Jefferson, L. Kabir, B. Mosk, and I.-S. Yang, Phys. Rev. D 91, 086013 (2015).

[14] R. Jefferson, SciPost Phys. 6, 042 (2019).

[15] K. Papadodimas and S. Raju, Phys. Rev. Lett. 112, 051301 (2014).

[16] K. Papadodimas and S. Raju, Phys. Rev. D 89, 086010 (2014).

[17] K. Papadodimas, arXiv:1708.06328.

[18] J. De Boer, S. F. Lokhande, E. Verlinde, R. Van Breukelen, and K. Papadodimas, J. High Energy Phys. 05 (2019) 010.

[19] M. A. Nielsen, arXiv:quant-ph/0502070.

[20] M. A. Nielsen, M. R. Dowling, M. Gu, and A. M. Doherty, Science 311, 1133 (2006).

[21] M. A. Nielsen and M. R. Dowling, arXiv:quant-ph/0701004.

[22] R. Jefferson and R. C. Myers, J. High Energy Phys. 10 (2017) 107.

[23] S. Chapman, M. P. Heller, H. Marrochio, and F. Pastawski, Phys. Rev. Lett. 120, 121602 (2018).

[24] J. Haegeman, T. J. Osborne, H. Verschelde, and F. Verstraete, Phys. Rev. Lett. 110, 100402 (2013).

[25] M. Nozaki, S. Ryu, and T. Takayanagi, J. High Energy Phys. 10 (2012) 193.

[26] A. Mollabashi, M. Nozaki, S. Ryu, and T. Takayanagi, J. High Energy Phys. 03 (2014) 098.

[27] L. Hackl and R. C. Myers, J. High Energy Phys. 07 (2018) 139.

[28] R. Khan, C. Krishnan, and S. Sharma, Phys. Rev. D 98, 126001 (2018).

[29] H. A. Camargo, P. Caputa, D. Das, M. P. Heller, and R. Jefferson, Phys. Rev. Lett. 122, 081601 (2019).

[30] S. Chapman, J. Eisert, L. Hackl, M. P. Heller, R. Jefferson, H. Marrochio, and R. C. Myers, SciPost Phys. 6, 034 (2019).

[31] M. Guo, J. Hernandez, R. C. Myers, and S.-M. Ruan, J. High Energy Phys. 10 (2018) 011.

[32] A. Bhattacharyya, A. Shekar, and A. Sinha, J. High Energy Phys. 10 (2018) 140.

[33] J. Cotler, M. R. Mohammadi Mozaffar, A. Mollabashi, and A. Naseh, arXiv:1806.02831.
[34] V. Balasubramanian, M. DeCross, A. Kar, and O. Parrikar, J. High Energy Phys. 02 (2019) 069.

[35] M. Miyaji, T. Takayanagi, and K. Watanabe, Phys. Rev. D 95, 066004 (2017).

[36] P. Caputa, N. Kundu, M. Miyaji, T. Takayanagi, and K. Watanabe, Phys. Rev. Lett. 119, 071602 (2017).

[37] P. Caputa, N. Kundu, M. Miyaji, T. Takayanagi, and K. Watanabe, J. High Energy Phys. 11 (2017) 097.

[38] G. Vidal, Phys. Rev. Lett. 101, 110501 (2008).

[39] A. Milsted and G. Vidal, arXiv:1807.02501.

[40] A. Milsted and G. Vidal, arXiv:1812.00529.

[41] B. Czech, Phys. Rev. Lett. 120, 031601 (2018).

[42] A. M. Polyakov, Phys. Lett. 103B, 207 (1981).

[43] See Supplemental Material at http://link.aps.org/ supplemental/10.1103/PhysRevLett.123.011601 for further discussions of coordinate transformations and norm properties.

[44] G. Evenbly and G. Vidal, Phys. Rev. Lett. 115, 180405 (2015).

[45] G. Vidal, Phys. Rev. Lett. 99, 220405 (2007).

[46] G. Evenbly and G. Vidal, Phys. Rev. Lett. 115, 200401 (2015).

[47] T. Takayanagi, J. High Energy Phys. 12 (2018) 048.

[48] C. V. Johnson, D-branes, Cambridge Monographs on Mathematical Physics (Cambridge University Press, Cambridge, England, 2005).

[49] M. Miyaji, T. Numasawa, N. Shiba, T. Takayanagi, and K. Watanabe, Phys. Rev. Lett. 115, 261602 (2015).

[50] A. Belin, A. Lewkowycz, and G. Sarosi, Phys. Lett. B 789, 71 (2019).

[51] A. Belin, A. Lewkowycz, and G. Sarosi, J. High Energy Phys. 03 (2019) 044.

[52] A. Bernamonti, F. Galli, J. Hernandez, R. C. Myers, S.-M. Ruan, and J. Simon, arXiv:1903.04511.

[53] P. Caputa and J. M. Magan, Phys. Rev. Lett. 122, 231302 (2019).

[54] J. M. Magan, J. High Energy Phys. 09 (2018) 043.

[55] C. A. Agon, M. Headrick, and B. Swingle, J. High Energy Phys. 02 (2019) 145. 